\title{
Surface finishing achieved by ECM with a moving wire electrode
}

\author{
Kohta Furuyama*, Masanori Kunieda*, Takashi Hashimoto**, Takashi Yuzawa** \\ (Received Feb.18, 2016)
}

\begin{abstract}
*Department of Precision Engineering, The University of Tokyo, Tokyo 113-8656, Japan
**Mitsubishi Electric
\end{abstract}

\section{Abstract}

It is known that good surface roughness can be achieved by ECM with a high current density. However, when the tool electrode moves across the surface of the workpiece the low current density area which follows the high current density area roughens the surface. Attempts to suppress the effect of the low current density area by utilizing pulse currents to obtain a glossy surface were made. This paper reports the experimental results of ECM with a moving wire electrode on an EDMed surface to investigate the effect of various conditions on the machined surface.

\section{INTRODUCTION}

Electrochemical machining (ECM) is a machining method that utilizes anodic dissolution to remove metals. As the basic principle of ECM is an electrochemical process, the machined surface shows no cracks, burrs, residual stress, nor any heat affected zones that are found in mechanical or thermal machining methods. In addition, it is known that when high current density is utilized in ECM, the machined surface has a low surface roughness with that confers a glossy texture [1]. Due to the above machining characteristics, ECM is utilized as a surface finishing method in several fields to achieve mirror-like surfaces free from machining damage [2]. To utilize ECM as a surface finishing method for free three dimensional surfaces, it is crucial to conduct machining while moving the tool electrode across the surface. However, success has proven elusive due to the effect of low current density roughening.

In ECM, with a low current density the surface tends to be non-lustrous [3]. The reason for rough non-lustrous surfaces obtained by low-current densities may be due to the uneven distribution of micro structures, defects and impurities which have different electrochemical potentials that result in variable rates of dissolution. In ECM, although most of the current that flows between the two electrodes concentrate at the smallest gap, a considerable amount will flow through the sides where the gap is large. This flow of electric current is called stray current. The current density distribution is highest at the smallest gap distance, gradually declining to zero at points further with distance. This creates low current density areas at the periphery of the high current density area, and as the tool electrode moves, it is inevitably machined by this low current density area [Fig.1]. In this process, the final machined surface is affected by the low current density area, and normally has a larger surface roughness compared to stationary machining with the same current, and also does not show glossiness across the whole surface but only at the point where the tool electrode halts [4]. Therefore, to utilize ECM as a scanning surface finishing method, it is necessary to limit the effect of machining within the zone of low current density areas.

EJM is an adaption of ECM $[5,6]$ that utilizes a jet stream of electrolyte ejected from a nozzle to the workpiece as a voltage is applied between the nozzle and the workpiece. The electrolyte jet creates a hydraulic jump, which restricts the spread of stray current. In electrolyte jet machining (EJM), it is possible to achieve a lustrous surface across the whole scanned surface by using a suitable pulse condition [7]. As EJM shares the same basic principles with ECM, the finishing methods used in EJM might be effective in ECM finishing with simple shaped electrodes such as wire electrodes.

The aim of this study was to investigate the ECM process with a moving wire electrode under conditions similar to the finishing methods used in EJM, in order to realize ECM surface finishing on free three dimensional surfaces.

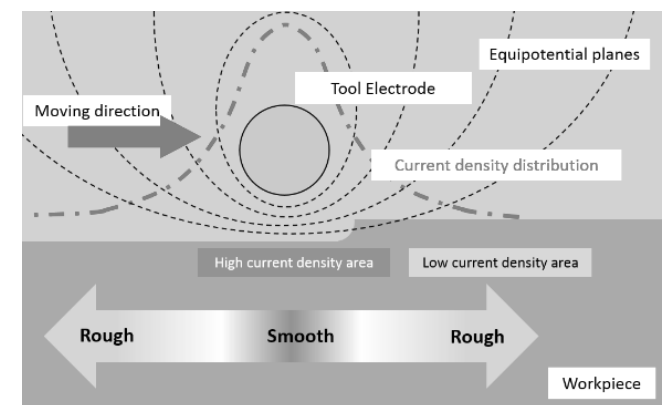

Fig.1 Problems of surface finishing with a moving cathode

\section{PRINCIPLES OF RESTRICTING MACHINING TO THE HIGH CURRENT DENSITY ZONE}

\subsection{Finishing method in EJM with a moving nozzle}

The surface finishing method of EJM is achieved by reducing the dissolution at low current density areas and limiting the machining to only the high current density area. This principle is reported to be the same as the theory of ultra short pulse ECM which utilizes the difference of the time required to form electric double layers [8]. Formation of electric double layers occur due to the behavior of ions in the electrolyte, and can be understood as the charging of capacitors as shown in Fig.2. Therefore, the formation of electric double layers take less time at the high current density area compared to the low current density area. This suggests that during a short time from when a voltage is applied, there exists a time range where the dissolution occurs only 
at the high current density area while at the low current density zone the current is charging the capacitor. Therefore, by applying voltage to the gap for a short time, the machining can be limited to the high current density area. By using pulse electric currents, the machining can be limited to the high current density area for each pulse voltage. However, if the capacitor at the low current density area is still partially charged from the previous pulse voltage, the time difference will not be large enough to reduce the dissolution at the low current density area. Therefore, it is crucial to discharge the capacitance of the electric double layers and return the interelectrode gap environment to an electrochemically neutral state between each pulse current. This is achieved by setting extremely long intervals between each pulse current, or by forcing the deformation of the electric double layers by inserting a reversed polarity pulse current. By using such pulse conditions in EJM, as the dissolution of the low current density area is reduced, a lustrous surface can be achieved across the whole surface when the nozzle moves over the workpiece [7].

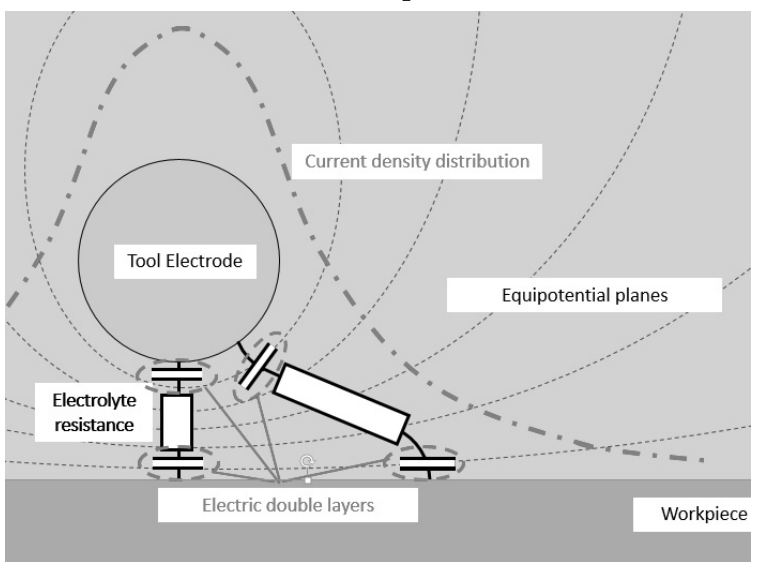

Fig.2 Equivalent circuit in ECM

\subsection{Comparison of current density distribution between EJM and ECM}

As EJM utilizes the hydraulic jump to limit the stray current, the roughening effect of the low current density area in scanning machining is naturally small. On the other hand, in conventional ECM, the stray current is larger, and is thought to create a larger roughening effect. To investigate the difference of the current density distribution between EJM and conventional ECM, computer simulations were conducted on COMSOL Multiphysics, a simulation software based on finite element analysis. The 2 -dimensional cross section of a wire electrode and a plate workpiece placed horizontally was used for the simulation model as shown in Fig.3. The wire diameter was normalized as $2 \mathrm{a}$, and the inter-electrode gap was set at two different distances $2 \mathrm{a}$ and $0.4 \mathrm{a}$ to investigate the influence of the gap on the current density distribution. The equipotential planes acquired when the wire electrode was set at $0 \mathrm{~V}$ and the workpiece at $15 \mathrm{~V}$ are shown in Fig.4, which clearly show the larger electric potential gradient at the smaller inter-electrode gap. From the acquired equipotential planes, the current density distribution was calculated. To compare the current density distribution of EJM and ECM with a wire electrode, the peak of the current density distribution and tool width were set uniform in Fig.5. When the gap in ECM was equal to the wire diameter, the current density distribution extends in a wider area than EJM, suggesting a stronger roughening effect of the low current density area as the tool electrode passes over the workpiece. In comparison, when the tool gap width is small, the current density distribution becomes narrower, which leads to machining limitation to the high current density zone. Therefore, it can be concluded that in the case of conventional ECM, it is essential to narrow the inter-electrode gap, opposed to EJM, which its current density distribution is independent of the gap distance between the nozzle electrode and the workpiece, as far as the electrolytic current is controlled to be constant.

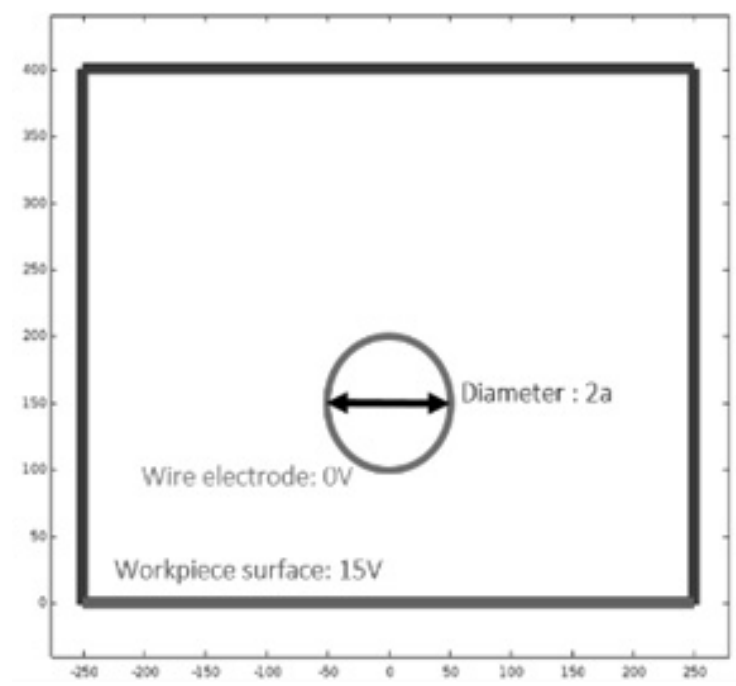

Fig.3 Simulation model of wire electrode and workpiece

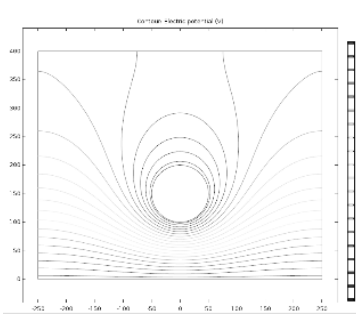

a) Gap $2 \mathrm{a}$

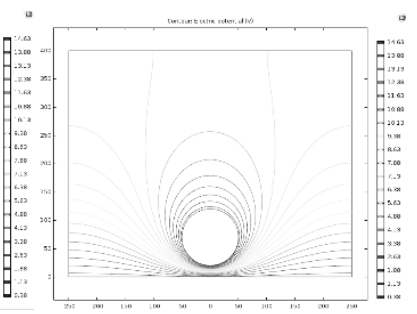

b) Gap $0.4 a$
Fig.4 Equipotential planes 


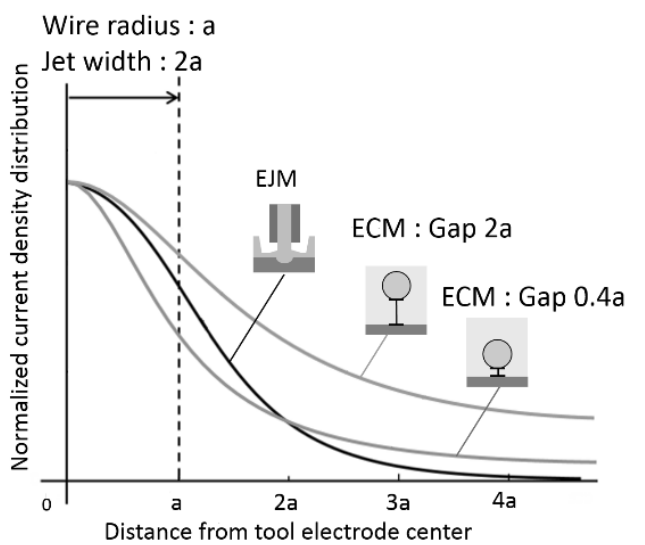

Fig.5 Comparison of current density distribution

\section{EXPERIMENTAL PROCEDURE}

A schematic view of the experimental equipment is shown in Fig.6. The wire electrode is fixed by the wire holder as shown in Fig.7. The workpiece is set to an orthogonal-type robot that can be controlled numerically for each axis, XYZ. The wire electrode reciprocates across the surface of the workpiece at a fixed speed of $0.5 \mathrm{~mm} / \mathrm{s}$ while machining, changing direction for each stroke of $5.0 \mathrm{~mm}$. Stainless steel (SUS 304) was used for the workpiece. The initial workpiece surface was machined by EDM, with surface roughness of Ra: $2.0 \mu \mathrm{m} . \mathrm{NaNO}_{3} 20 \%$ aqueous solution is emitted from a nozzle to supply electrolyte between the two electrodes and to flush away impurities such as bubbles and sludge that are formed simultaneously with machining. The electrolyte is filtered and returned to the electrolyte tank after machining, and is used repeatedly to realize continuous machining. Machining current is supplied by a high speed bipolar power supply that amplifies the pulse voltage created by a function generator. Machining current can be controlled as either constant current or constant voltage.

A surface finishing method can be evaluated by four performances. The surface roughness and gloss of the machined surface, the undercut of the machined edge, and the machining depth required to improve the surface. Surface roughness and gloss are the performances to be improved which affect the functionality and design of the final product. Glossiness is the optical property of a surface which indicates how well it reflects light in mirror-like direction. A glossmeter is used to measure the gloss of a surface by projecting a beam of light at a fixed angle, and by measuring the amount of reflected light at the opposite equal angle, as shown in Fig.8. For this research, a digital glossmeter (Suga Test Instruments) was utilized for gloss measurement. Undercut and machined depth are performances that inevitably occur upon finishing, but negatively affect the precision of the product. Therefore, conditions that improve the surface without a large machined depth and undercut are preferable. In this research, the effect of the machined depth were also taken into account to compare different conditions. The undercut of the machined surface was observed by SEM images.

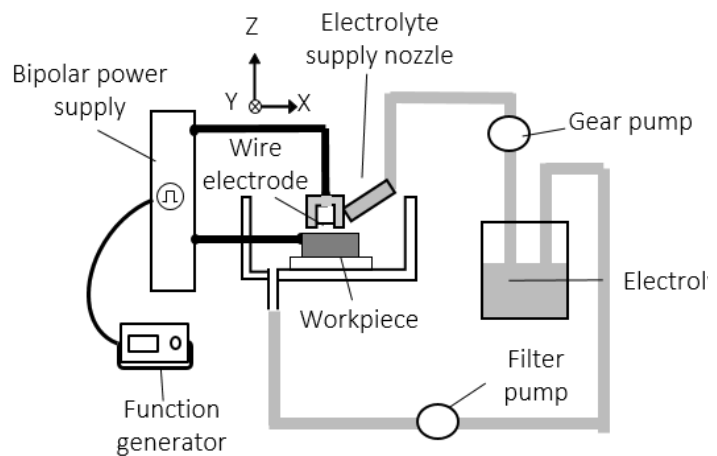

Fig.6 Experimental equipment

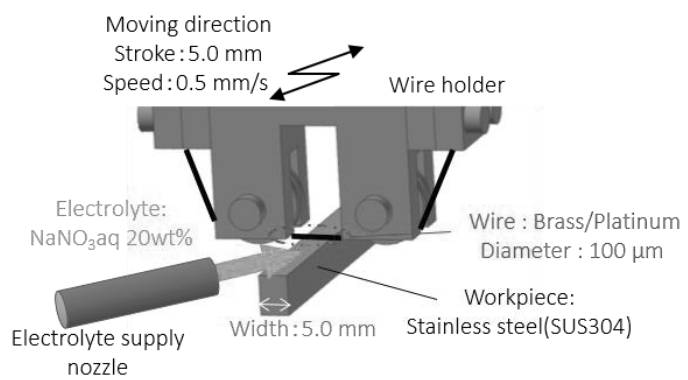

Fig.7 Wire electrode and workpiece

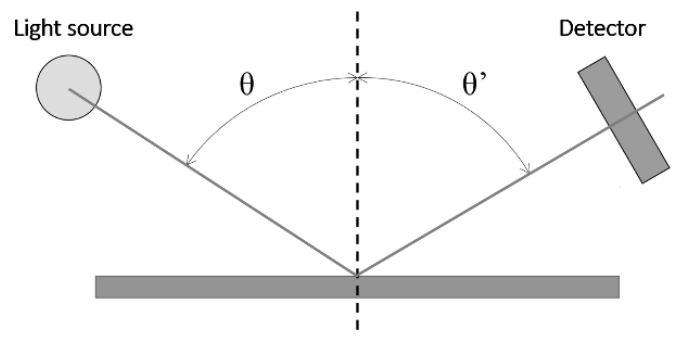

Fig. 8 Schematic view of a glossmeter

\section{FINISHING WITH MONOPOLAR PULSE CURRENT}

\subsection{Effect of the inter-electrode gap}

The effect of the gap width on the surface roughness was investigated using pulse currents. Constant current controlled pulse currents were used. Machining conditions are shown in Table 1. To investigate how the surface roughness improves with the machined depth, experiments were conducted several times with different machining times for each condition. As shown in Fig.9, when the gap is smaller, the machining speed is faster. From Fig.10, it can be seen that when the gap is smaller, the surface roughness improves at shallower machining depths, and the convergent surface roughness is also lower. This supports the hypothesis that a narrow gap is important to achieve low surface roughness. However, glossy surfaces could not be achieved. 
Table 1 Machining conditions to investigate the effect of inter-electrode gap

\begin{tabular}{l|c|c}
\hline Pulse duration $[\mu \mathrm{s}]$ & \multicolumn{2}{|c}{10} \\
\hline Pulse period $[\mu \mathrm{s}]$ & \multicolumn{2}{|c}{100} \\
\hline Duty factor $[\%]$ & \multicolumn{2}{|c}{10} \\
\hline Inter-electrode gap $[\mu \mathrm{m}]$ & 100 & 20 \\
\hline Wire & \multicolumn{2}{|c}{ Brass } \\
\hline Wire diameter $[\mu \mathrm{m}]$ & 100 \\
\hline
\end{tabular}

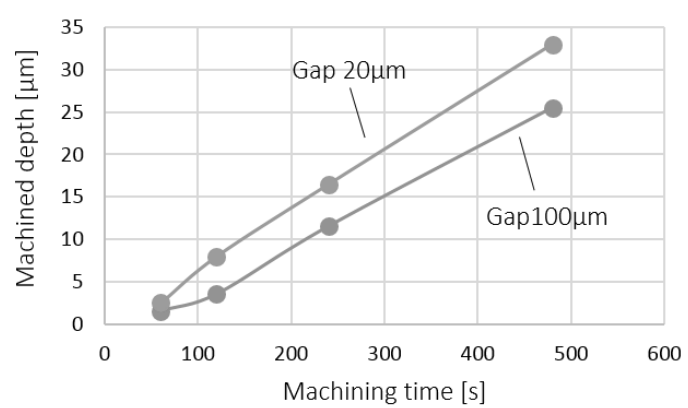

Fig.9 Effect of gap on relationship between machining time and machined depth

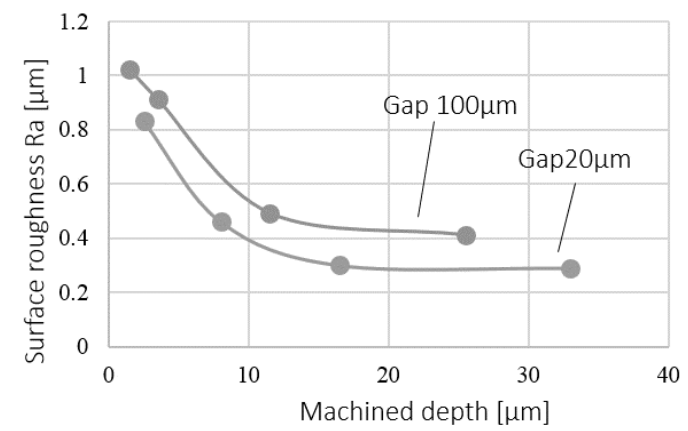

Fig.10 Effect of gap on relationship between machined depth and surface roughness

\subsection{Effect of the duty factor}

The effect of the duty factor on surface roughness and glossiness was investigated using conditions shown in Table 2. For comparison, direct current was also used. Experiment results are shown in Fig.11. By using pulse currents, the surface roughness of the final machined surface improves effectively compared to direct current machining. When the duty factor is as small as $1 \%$, the surface roughness converges at a shallower depth than pulse conditions with larger duty factors. In addition, the surfaces of converged surface roughness showed glossiness at duty factor $1 \%$. However, the current efficiency of machining is lower than other conditions with a pulse current of duty factor $1 \%$. The horizontal axis of Fig.12 is the total current that flows through the inter-electrode gap during machining, and the vertical axis shows the machined depth. It can be seen that with the same electric charge flowing through the interelectrode gap, the machined depth is significantly shallower for the pulse current of duty factor $1 \%$. This is thought to be that at duty factor $1 \%$, the electric double layers are completely returned to neutral state, and more electric charge is required to form them repeatedly at each pulse. This results in less energy used for machining, which results in a lower current efficiency. As the duty factor also affects the machining speed, when the duty factor is $1 \%$ the machining time required to achieve the same machined depth is extremely long compared to other conditions as shown in Fig.13.

Table 2 Machining conditions to investigate the effect of the duty factor

\begin{tabular}{l|c|c|c|c}
\hline Pulse duration $[\mu \mathrm{s}]$ & \multirow{3}{*}{ D.C. } & \multicolumn{3}{|c}{100} \\
\cline { 3 - 5 } Pulse period $[\mu \mathrm{s}]$ & 500 & 1000 & 10000 \\
\cline { 3 - 5 } & & 20 & 10 & 1 \\
\hline $\begin{array}{l}\text { Duty factor }[\%] \\
\text { Inter-electrode gap } \\
{[\mu \mathrm{m}]}\end{array}$ & \multicolumn{3}{|c}{20} \\
\hline
\end{tabular}

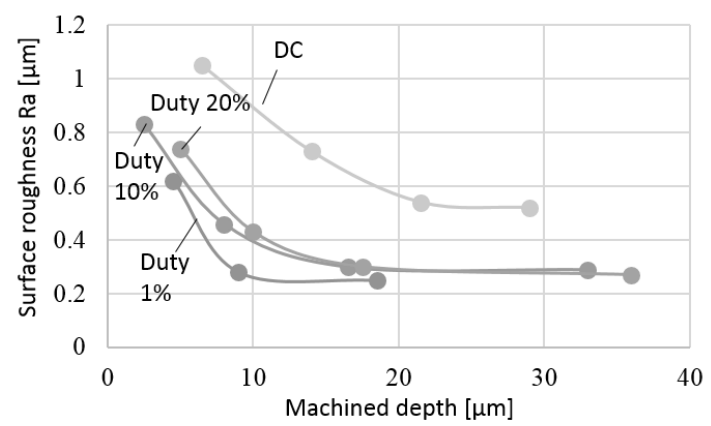

Fig.11 Effect of duty factor on relationship between machined depth and surface roughness

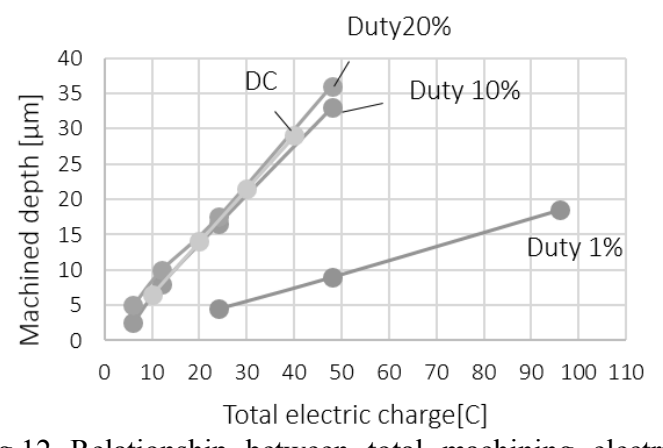

Fig.12 Relationship between total machining electric charge and machined depth with different duty factors

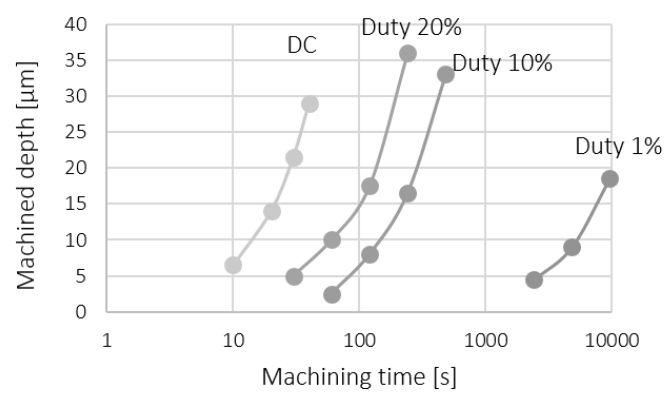

Fig.13 Relationship between total machining electric charge and machined depth with different duty factors 


\subsection{Evaluation of glossiness}

It was found that the machined surface shows glossiness when a pulse current of duty factor $1 \%$ was used. The glossiness of the machined surface at duty factor $1 \%$ and $10 \%$ are compared at Fig.14. Although the converged surface roughness is about the same as shown in Fig.11, the glossiness at deep machined depths differ significantly. It is known that in the case of metals, glossiness is strongly affected by the average slope of the surface roughness curve. By comparing the surface roughness curve of the deepest machined surfaces of both conditions duty factor $10 \%$ and $1 \%$ shown in Fig. 15 , it can be clearly seen that the average slope of the surface roughness is larger at duty $10 \%$, which is thought to be the reason for the non-lustrous surface.

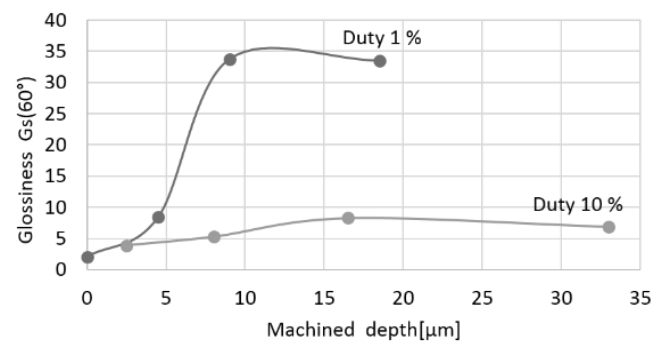

Fig.14 Effect of duty factor on relationship between machined depth and glossiness

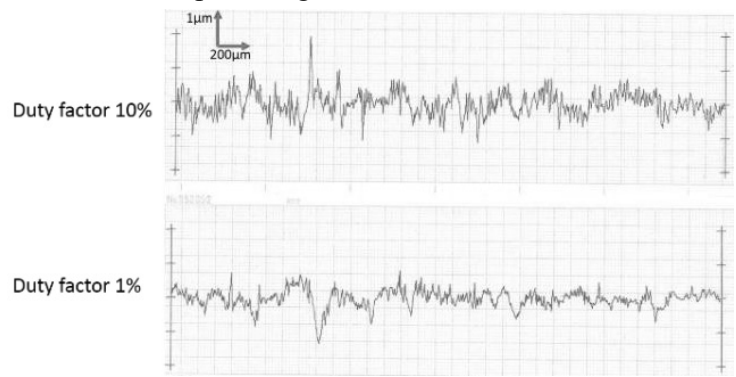

Fig.15 Comparison of surface roughness curve for duty factor $10 \%$ and $1 \%$

\section{FINISHING WITH BI-POLAR PULSE CURRENT}

\subsection{Pulse conditions}

It was found that by using a narrow gap and a pulse current with a low duty factor, it is possible to achieve a machined surface with low surface roughness and a glossy texture across the whole machined pass. However, the time required for machining is extremely long and impractical. In order to achieve shorter machining time, bi-polar pulse currents were used in EJM. To investigate the effect of bipolar pulse currents with ECM using a wire electrode, machining experiments were conducted. Constant voltage controlled pulse currents were used for machining. The pulse shape is shown in Fig. 16. To avoid the dissolution of the wire electrode when the wire electrode is the anode, platinum wire was used as the wire electrode for bi-polar pulse conditioned machining.

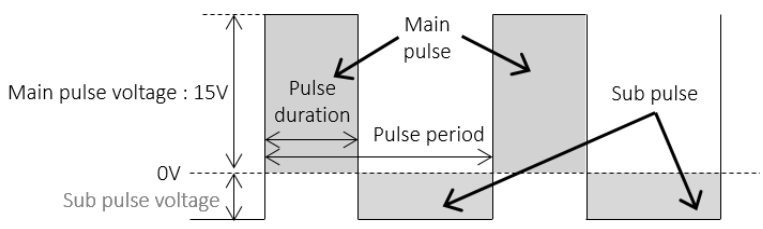

Fig16. Pulse voltage shape

\subsection{Effect of sub pulse voltage at duty $10 \%$}

Experimental conditions are shown in Table 3. For comparison, positive voltage were also used as well as negative voltage for the sub pulse. The electric charge that flows through the gap per sub pulse was measured, which results are shown in Fig.17. Although the sub pulse was at 0 $\mathrm{V}$, opposite current occurred. This is thought to be caused by the concentration cell that is formed between the two electrodes when the main pulse is applied. As shown in Fig.18, it is found that as the sub pulse voltage becomes larger in the negative, the depth required to achieve low surface roughness becomes larger. When the absolute of the negative sub voltage is larger than $1.0 \mathrm{~V}$, the surface roughness increases with the machined depth. For other conditions, the converged surface roughness is about the same. However, the absolute slope of the roughness curve and the gloss of the surface was significantly different depending on the sub pulse voltage. The glossiness of the deepest machined surface for each sub pulse voltage is shown in Fig. 19. The machined surface showed the highest gloss at sub pulse voltage $-0.5 \mathrm{~V}$. The relationship between the reciprocal of the average absolute slope and gloss were found to be proportional as shown in Fig.20. It can be concluded that by using a suitable condition, finished surfaces with low surface roughness and small average absolute slope that result in a glossy texture can be achieved with bi-polar pulse currents.

Table 3 Machining conditions with bi-polar pulse currents

\begin{tabular}{|c|c|c|c|c|c|c|}
\hline Main pulse voltage [V] & \multicolumn{6}{|c|}{15} \\
\hline Sub pulse voltage $[\mathrm{V}]$ & -2.0 & -1.0 & -0.5 & 0 & 2.0 & 3.0 \\
\hline Main pulse period $[\mu \mathrm{s}]$ & \multicolumn{6}{|c|}{100} \\
\hline Pulse period $[\mu \mathrm{s}]$ & \multicolumn{6}{|c|}{900} \\
\hline Duty factor $[\%]$ & \multicolumn{6}{|c|}{10} \\
\hline Inter-electrode gap $[\mu \mathrm{m}]$ & \multicolumn{6}{|c|}{20} \\
\hline Wire & \multicolumn{6}{|c|}{ Platinum } \\
\hline Wire diameter $[\mu \mathrm{m}]$ & \multicolumn{6}{|c|}{100} \\
\hline Number of strokes & 2 & & 4 & $\varepsilon$ & & 16 \\
\hline
\end{tabular}

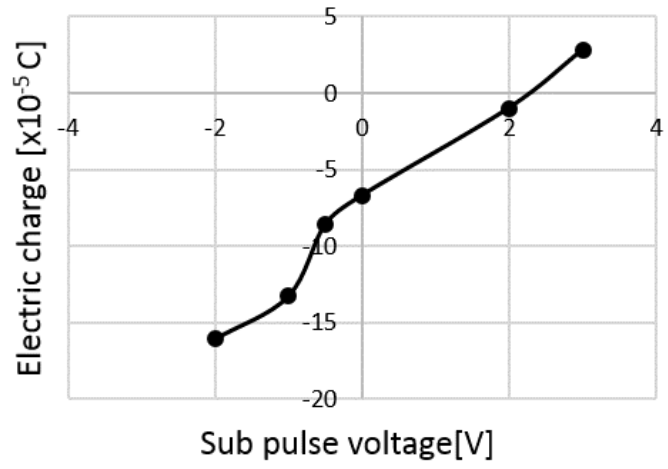

Fig.17 Relationship between sub pulse voltage and electric charge 


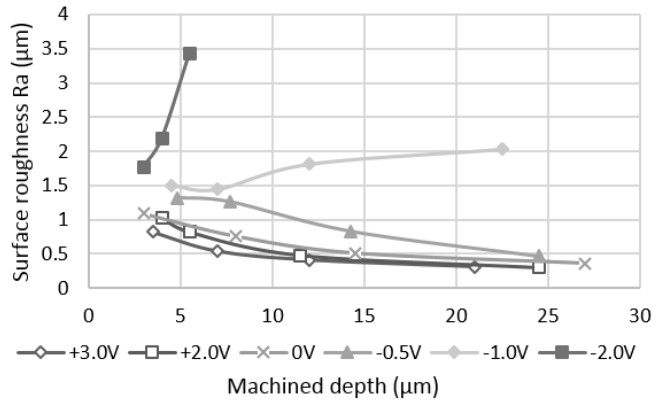

Fig.18 Effect of sub pulse voltage on relationship between machined depth and surface roughness

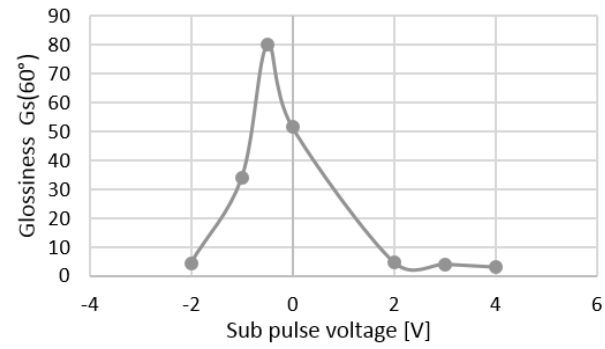

Fig.19 Relationship between sub pulse voltage and glossiness

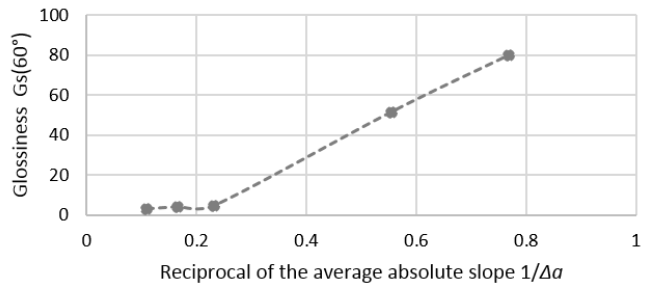

Fig.20 Relationship between reciprocal of average absolute slope and glossiness

\subsection{Effect of sub pulse voltage at duty $40 \%$}

It was found that by using bipolar pulse currents glossy surfaces could be created at a higher duty factor than monopolar pulse currents. To investigate the possibility of bipolar pulse finishing at even higher duty factors, experiments were conducted at duty factor $40 \%$. Experimental conditions are shown in Table 4. As shown in Fig.21, it is found that as the sub pulse voltage becomes larger in the negative, the depth required to achieve low surface roughness becomes larger. This tendency was also found in bipolar finishing at duty $10 \%$. The glossiness of the deepest machined surface for each sub pulse voltage is shown in Fig.22. The machined surface showed the highest gloss at sub pulse voltage $-4.0 \mathrm{~V}$. The SEM images of the machined surfaces of sub pulse voltage $0 \mathrm{~V}$ and $-4.0 \mathrm{~V}$ are compared in Fig.23. From the SEM images, it can be seen that at sub voltage $0 \mathrm{~V}$, microscopic roughness can be found. From Fig. 24 it can be clearly seen that the machined surface with a sub pulse voltage of $-4.0 \mathrm{~V}$ has a high gloss compared to that of $0 \mathrm{~V}$. The microscopic roughness is thought to create the large average absolute slope, which results in a nonlustrous surface. The SEM image of the edge of the machined surface is shown in Fig.25. It can be seen that the undercut of the machined surface is not large. Hence, the edge is not dulled significantly.
Table 4 Machining conditions with duty of $40 \%$

\begin{tabular}{l|c|c|c|c|c}
\hline Main pulse voltage [V] & \multicolumn{5}{|c}{15} \\
\hline Sub pulse voltage [V] & -6.0 & -4.0 & -2.0 & -1.0 & 0 \\
\hline Main pulse period $[\mu \mathrm{s}]$ & \multicolumn{5}{|c}{100} \\
\hline Pulse period $[\mu \mathrm{s}]$ & \multicolumn{5}{|c}{250} \\
\hline Duty factor [\%] & \multicolumn{5}{|c}{20} \\
\hline Inter-electrode gap [ $\mu \mathrm{m}]$ & \multicolumn{5}{|c}{20} \\
\hline Number of strokes & 1 & 2 & 4 & 8 \\
\hline
\end{tabular}

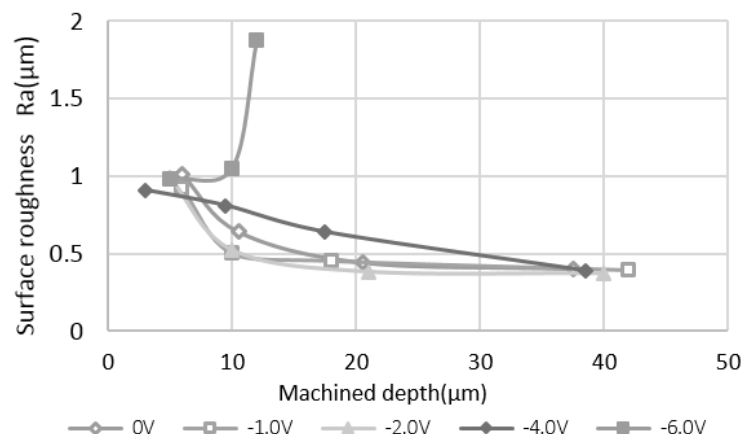

Fig.21 Effect of sub pulse voltage on relationship between machined depth and surface roughness

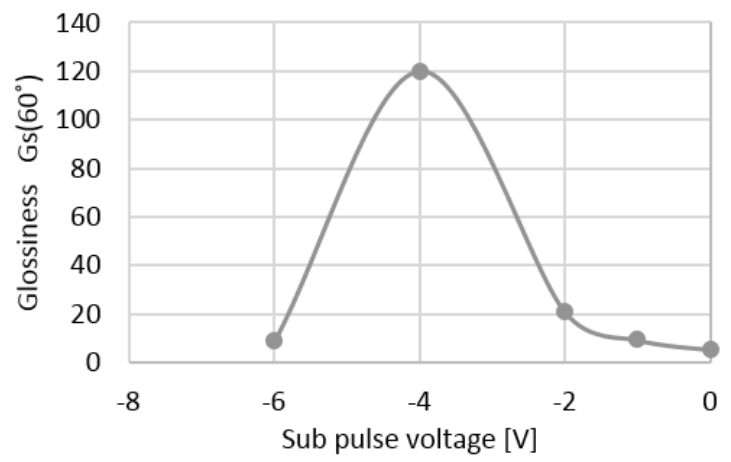

Fig.22 Relationship between sub pulse voltage and glossiness
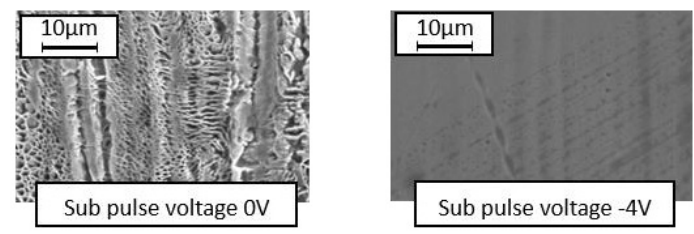

Fig.23 Comparison of SEM images of machined surfaces at sub pulse voltage $0 \mathrm{~V}$ and $-4.0 \mathrm{~V}$
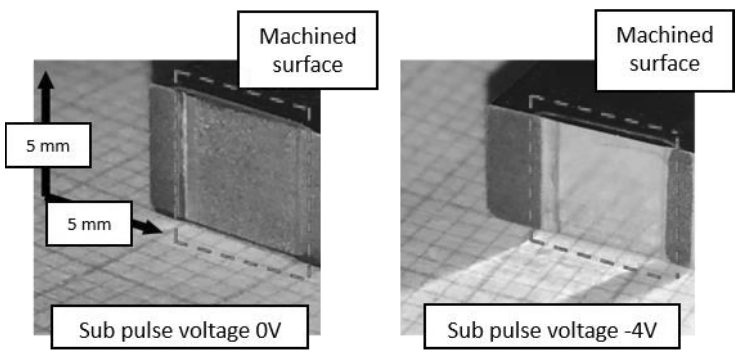

Fig.24 Comparison of SEM images and photos of machined surfaces at sub pulse voltage $0 \mathrm{~V}$ and $-4.0 \mathrm{~V}$ 


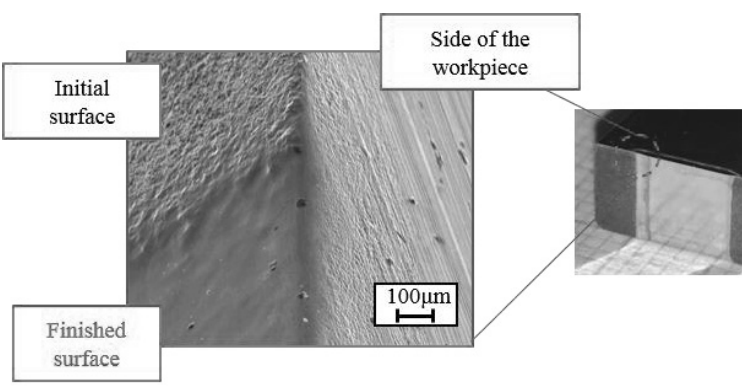

Fig.25 SEM image of machined edge at sub pulse voltage $-4.0 \mathrm{~V}$

\section{CONCLUSIONS}

Finishing machining with ECM using a wire electrode was conducted using various conditions. It was found that by setting a small inter-electrode gap, finishing methods utilized in EJM can also be used in ECM with a wire electrode to achieve a glossy surface across the area where the cathode moves. With monopolar pulse currents, glossy surfaces could be achieved by using pulse conditions of duty factor $1 \%$, but with the drawback of an extremely long machining time. By using bipolar pulse currents, glossy surfaces with low surface roughness could be obtained at both duty factors $10 \%$ and $40 \%$. By using a bipolar pulse current of duty factor $40 \%$, with the main pulse voltage set at $15 \mathrm{~V}$ and the sub pulse voltage set at $-4 \mathrm{~V}$, the initial surface roughness Ra $2.0 \mu \mathrm{m}$ was reduced to $0.39 \mu \mathrm{m}$, and showed high glossiness with a short machining time. These results contribute to the realization of ECM surface finishing with a moving tool electrode.

The pulse conditions used in the experiments had very long pulse durations of $100 \mu$ s compared to ultra short pulse ECM which utilizes pulse durations of 50 200 nanoseconds. Therefore, the principle of the machining limitation may not be fully explained by the charge and discharge of electric double layers. A more accurate understanding of the phenomena may be the formation of concentration boundary layers that form when voltage is applied. The formation of concentration boundary layers can also be understood as capacitors, and may have a longer charging time compared to double layers. Further research is expected to be conducted in future.

\section{Acknowledgements}

This work was supported by the Cross-Ministerial Strategic Innovation Promotion Program (SIP): Innovative Design/ Manufacturing Technologies, funded by NEDO.

\section{References}

[1] Konig, W., Lindenlauf, P., 1978, Surface Generation in Electrochemical Machining, Annals of the CIRP, 27, 1: 97100

[2] Rajurkar KP, Zhu D, McGeough JA, Kozak J, De Silva A (1999) New Developments in Electro-Chemical Machining, Annals of the CIRP 48(2):567-579.

[3] T. Masuzawa, S. Sakai, Quick finishing of WEDM products by ECM using a mate electrode, Annals of the CIRP 36 (1) (1987) 123-126.

[4] Masuzawa, T., Sakai, S., 1983, Wire-ECM Finishing Surfaces Produced by NC-Wire EDM, Proc. of ISEM 7: 285291

[5] Ippolito R, Tornincasa S, Capello G (1981) Electron-Jet Drilling. Annals of the CIRP 30(1):87-89.

[6] Kozak J (1989) Some Aspects of Electro Jet Drilling. 4th International Conference on Developments in Production Engineering Design \& Control, 363-369.

[7] Takuma Kawanaka, Masanori Kunieda: Mirror-like finishing by electrolyte jet machining, CIRP Annals64,1 237-240

[8] Schuster R, Kircher V, Allonfue P, Etrl F (2000) Electrochemical Micromachining. Science 289(5476):98101. 